Supporting Information for

\title{
Morphological transitions of a photoswitchable aramid amphiphile nanostructure
}

Dae-Yoon Kim ${ }^{1,2 \dagger}$, Ty Christoff-Tempesta ${ }^{1 \dagger}$, Guillaume Lamour ${ }^{3}$, Xiaobing Zuo ${ }^{4}$, Ki-Hyun Ryu ${ }^{2}$, Julia H. Ortony ${ }^{*}$

${ }^{1}$ Department of Materials Science and Engineering, Massachusetts Institute of Technology, Cambridge, MA 02139, USA.

${ }^{2}$ Institute of Advanced Composite Materials, Korea Institute of Science and Technology, Bondong, JB 55324, Korea.

${ }^{3}$ LAMBE, Université Paris-Saclay, University of Evry, CNRS, Evry-Courcouronnes, France

${ }^{4}$ X-ray Science Division, Advanced Photon Source, Argonne National Laboratory, Argonne, IL 60439, USA.

$\dagger$ These authors contributed equally.

*Correspondence to: ortony@mit.edu.

\section{Contents:}

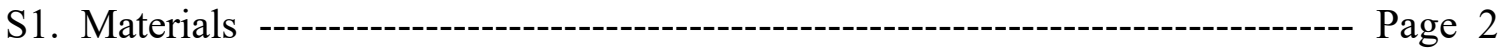

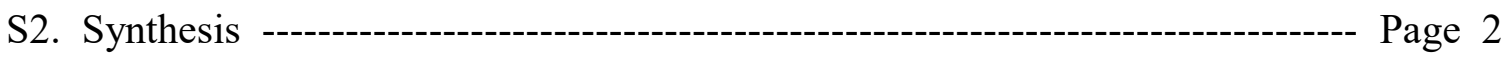

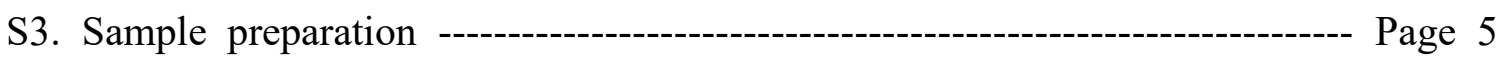

S4. A note on vinylnitrile isomer naming conventions ----------------------- Page 5

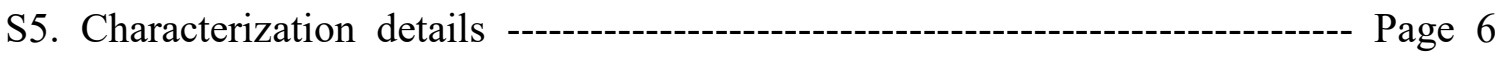

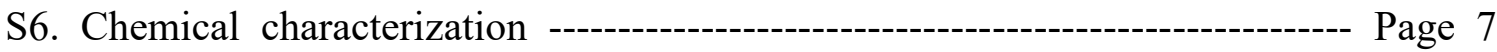

S7. Nanostructure fitting to small angle X-ray scattering profiles ----------- Page 12

S8. Statistical topographical analysis of nanoribbon contours -------------- Page 14

S9. UV-Vis spectroscopy controls and experiments------------------------------ Page 16

S10. Probing for the Tyndall effect in the trans and cis states of 2 ------ Page 18

S11. Packing of compound 2 into nanoribbons and nanotubes ------------- Page 19 


\section{S1. Materials}

Methyl 4-aminobenzoate (Sigma Aldrich, 98\%), 3,3-dimethylbutyric acid (Sigma Aldrich, 98\%), 4-aminobenzyl cyanide (Sigma Aldrich, 99\%), methyl 4-formylbenzoate (Sigma Aldrich, 99\%), 1,4-bis-Boc-1,4,7-triazaheptane (Chem Impex, 99\%), 1-ethyl-3-(3dimethylaminopropyl)carbodiimide hydrochloride (EDC, TCI Chemicals, 98\%), 4dimethylaminopyridine (DMAP, TCI Chemicals, 99\%), lithium hydroxide (LiOH, Alfa Aesar, 98\%), potassium hydroxide ( $\mathrm{KOH}$, Alfa Aesar, 98\%), hydrochloric acid ( $\mathrm{HCl}$, Alfa Aesar, 37\%), trifluoroacetic acid (TFA, Alfa Aesar, 99\%), diethylether (DEE, Acros Organics, 99\%), tetrahydrofuran (THF, Acros Organics, 99\%), ethyl acetate (EA, Acros Organics, 99\%), hexane (HEX, Acros Organics, 99\%), methanol (MeOH, Acros Organics, 99\%), ethanol (EtOH, Acros Organics, 99\%), dichloromethane (DCM, Acros Organics, 99\%), and dimethylformamide (DMF, Acros Organics, 99\%) were used as received without further purification.

\section{S2. Synthesis}

(A)

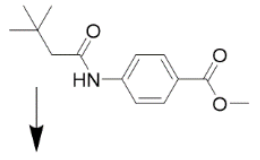

(B)

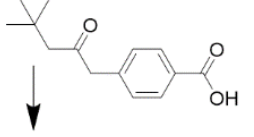

(C)

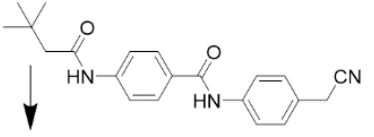

(D)

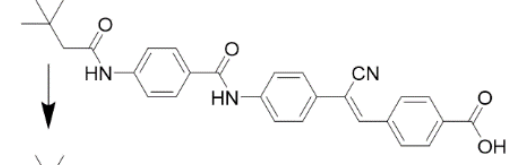

(E)

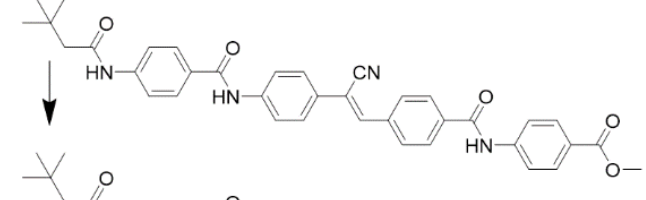

(F)

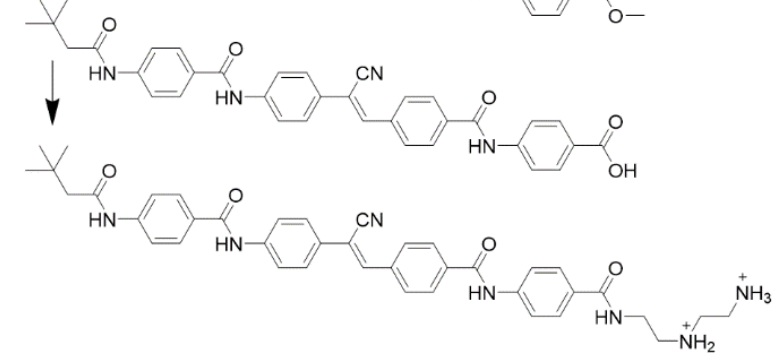

Figure S1. Synthetic route to obtain the photoresponsive aramid amphiphile 2. 
Methyl 4-(3,3-dimethylbutanamido)benzoate (compound A): A solution of methyl 4aminobenzoate (5.5 mmol), 3,3-dimethylbutyric acid (8.3 mmol), EDC (16.5 mmol), and DMAP (16.5 mmol) in DCM (40 mL) was stirred at room temperature for $24 \mathrm{~h}$. The solution was washed with deionized water after the reaction and then extracted in DCM. The organic layer was purified by performing column chromatography with silica gel using EA:HEX $=1: 1$ as eluent. Yield: 81\%. ${ }^{1} \mathrm{H}$ NMR (400 MHz, DMSO- $d$ ): $\delta=7.92(\mathrm{~d}, 2 \mathrm{H}), 7.73(\mathrm{~d}, 2 \mathrm{H}), 3.82(\mathrm{~s}$, $3 \mathrm{H}), 2.27(\mathrm{~s}, 2 \mathrm{H}), 1.07(\mathrm{~s}, 9 \mathrm{H}) \mathrm{ppm}$.

4-(3,3-Dimethylbutanamido)benzoic acid (compound B): $10 \mathrm{M} \mathrm{LiOH} \mathrm{(5} \mathrm{mL)} \mathrm{was}$ added to a stirred solution of compound $\mathbf{A}(2.1 \mathrm{mmol})$ in EtOH $(20 \mathrm{~mL})$. The mixture was heated to $70{ }^{\circ} \mathrm{C}$ for $6 \mathrm{~h}$, and then neutralized with $1 \mathrm{M} \mathrm{HCl}$ solution. The precipitate was obtained by filtration and washed with water several times. The crude product was purified by reprecipitation from chloroform and methanol, and then dried under vacuum. Yield: $99 \% .{ }^{1} \mathrm{H}$ NMR (400 MHz, DMSO- $d$ ): $\delta=7.87$ (d, 2H), $7.71(\mathrm{~d}, 2 \mathrm{H}), 2.23(\mathrm{~s}, 2 \mathrm{H}), 1.03(\mathrm{~s}, 9 \mathrm{H}) \mathrm{ppm}$.

$N$-(4-(cyanomethyl)phenyl)-4-(3,3-dimethylbutanamido)benzamide (compound $\mathbf{C}$ ): EDC (6.5 mmol) and DMAP (6.5 mmol) were added to a solution of compound $\mathbf{B}(2.1 \mathrm{mmol})$ in DMF $(20 \mathrm{~mL})$. After stirring at room temperature for $20 \mathrm{~min}$, 4-aminobenzyl cyanide (6.5 mmol) was added as a solid and the solution was sonicated in an ultrasound bath until a clear solution was obtained. The solution was stirred for $24 \mathrm{~h}$ at room temperature. A precipitate formed after addition of distilled water $(40 \mathrm{~mL})$, which was was collected by filtration and purified by washing with copious MeOH. Yield: 79\%. ${ }^{1} \mathrm{H}$ NMR (400 MHz, DMSO- $d$ ): $\delta=7.94$ $(\mathrm{d}, 2 \mathrm{H}), 7.84(\mathrm{~d}, 2 \mathrm{H}), 7.72(\mathrm{~d}, 2 \mathrm{H}), 7.34(\mathrm{~d}, 2 \mathrm{H}), 4.05$ (s, 2H), 2,27 (s, 2H), 1.03 (s, 9H) ppm.

(Z)-4-(2-cyano-2-(4-(4-(3,3-dimethylbutanamido)benzamido)phenyl)vinyl)benzoicacid (compound $\mathbf{D})$ : Compound $\mathbf{C}(1.6 \mathrm{mmol})$ and methyl 4-formylbenzoate $(1.8 \mathrm{mmol})$ were dissolved in EtOH $(40 \mathrm{~mL})$ and THF $(30 \mathrm{~mL})$. The solution was heated in reflux under a nitrogen atmosphere, and $\mathrm{KOH}(10.3 \mathrm{mmol})$ was carefully added. The reaction mixture was cooled to room temperature after $24 \mathrm{~h}$. $6 \mathrm{M} \mathrm{HCl}$ solution was added to form a yellow precipitate which was filtered and washed with DCM. Yield: 88\%. ${ }^{1} \mathrm{H}$ NMR (400 MHz, DMSO- $d$ ): $\delta=$ $8.07(\mathrm{~m}, 3 \mathrm{H}), 8.03$ (d, 2H), $7.95(\mathrm{~d}, 4 \mathrm{H}), 7.81(\mathrm{~d}, 2 \mathrm{H}), 7.75(\mathrm{~d}, 2 \mathrm{H}), 2.24(\mathrm{~s}, 2 \mathrm{H}), 1.04(\mathrm{~s}, 9 \mathrm{H})$ ppm.

Methyl (Z)-4-(4-(2-cyano-2-(4-(4-(3,3-dimethylbutanamido)benzamido)phenyl)vinyl)benzamido)benzoate (compound $\mathbf{E})$ : A solution of compound $\mathbf{D}(0.6 \mathrm{mmol})$ in DMF (20 $\mathrm{mL}$ ) was added to a solution of methyl 4 -aminobenzoate $(1.8 \mathrm{mmol})$, EDC (1.8 mmol), and 
DMAP $(1.8 \mathrm{mmol})$ in DCM $(20 \mathrm{~mL})$. After stirring the mixture for $24 \mathrm{~h}$ at room temperature, the solvent was evaporated under reduced pressure. The product was obtained after washing with $\mathrm{MeOH}$ and drying. Yield: 83\%. ${ }^{1} \mathrm{H}$ NMR (400 MHz, DMSO- $d$ ): $\delta=8.11(\mathrm{~m}, 3 \mathrm{H}), 8.06$ (d, 2H), 7.99 (m, 8H), $7.83(\mathrm{~d}, 2 \mathrm{H}), 7.75(\mathrm{~d}, 2 \mathrm{H}), 3.79$ (s, 3H), 2.24 (s, 2H), 1.05 (s, 9H) ppm.

(Z)-4-(4-(2-cyano-2-(4-(4-(3,3-dimethylbutanamido)benzamido)phenyl)vinyl)benzamido)benzoic acid (compound F): $10 \mathrm{M} \mathrm{LiOH}(10 \mathrm{~mL}$ ) was added to a stirred solution of compound $\mathbf{E}(1.5 \mathrm{mmol})$ in $\mathrm{EtOH}(30 \mathrm{~mL})$. The mixture was refluxed for $3 \mathrm{~h}$ and then neutralized with an $1 \mathrm{M} \mathrm{HCl}$ solution. The precipitate was obtained by filtration and washed with water several times. Yield: 98\%. ${ }^{1} \mathrm{H}$ NMR (400 MHz, DMSO- $d$ ): $\delta=8.13(\mathrm{~m}, 3 \mathrm{H}), 8.01$ (d, 2H), 7.97 (m, 8H), 7.83 (d, 2H), 7.74 (d, 2H), 2.25 (s, 2H), 1.04 (s, 9H) ppm.

Synthesis of (Z)-N1-(2-(4-(4-(2-cyano-2-(4-(4-(3,3-dimethylbutanamido)benzamido)phenyl)vinyl)benzamido)benzamido)ethyl)ethane-1,2-diaminium (2): Compound F (0.3 mmol), 1,4-bis-Boc-1,4,7-triazaheptane (0.6 mmol), EDC (0.9 mmol), and DMAP (0.9 mmol) were dissolved in DMF $(20 \mathrm{~mL})$. The mixture was stirred at $25^{\circ} \mathrm{C}$ for $72 \mathrm{~h}$. The solvent was evaporated under reduced pressure. After addition of water, the crude mixture was filtered and washed with EA. The precipitate was then dissolved in the solution of DCM $(20 \mathrm{~mL})$. The mixture was held at $0{ }^{\circ} \mathrm{C}$, and a solution of TFA $(5 \mathrm{~mL})$ in DCM $(5 \mathrm{~mL})$ was carefully added. The mixture was stirred for $3 \mathrm{~h}$. After evaporating solvents, the resultant solid was washed with THF, DEE, and DCM several times. Yield: 54\%.

Chemical characterization of $\mathbf{2}$ :

${ }^{1} \mathrm{H}$ NMR (400 MHz, DMSO- $d$ ): $\delta=8.13(\mathrm{~m}, 3 \mathrm{H}), 8.07$ (d, 2H), $7.98(\mathrm{~m}, 4 \mathrm{H}), 7.92(\mathrm{~m}$, 4H), $7.82(\mathrm{~d}, 2 \mathrm{H}), 7.75(\mathrm{~d}, 2 \mathrm{H}), 3.57$ (m, 2H), $3.19(\mathrm{~m}, 6 \mathrm{H}), 2.25(\mathrm{~s}, 2 \mathrm{H}), 1.05(\mathrm{~s}, 9 \mathrm{H})$ ppm.

${ }^{13} \mathrm{C}$ NMR (400 MHz, DMSO- $d$ ): $\delta=171.4,167.5,165.58,143.2,142.1,141.4,139.2$, $138.3,136.7,129.4,128.7,126.9,120.9,119.2,118.8,116.9,111.9,49.6,48.3,45.1$, $34.5,32.3,30.5 \mathrm{ppm}$.

MS (MALDI-ToF) m/z calculated: $687.84 ; \mathrm{m} / \mathrm{z}$ calculated for $[\mathrm{M}+\mathrm{Na}]^{+}$adduct: 710.83 ; $\mathrm{m} / \mathrm{z}$ found: 710.83 .

Elemental Analysis calculated (\%) for $\mathrm{C}_{40} \mathrm{H}_{45} \mathrm{~N}_{7} \mathrm{O}_{4}$ : C 69.85, H 6.59, N 14.25, O 9.30; found: C 69.84, H 6.57, N 14.24. 


\section{S3. Sample preparation}

Deionized water was added to a sample of $\mathbf{2}$ and a sonicator bath was used to promote self-assembly. After 24 h post-sonication at room temperature, 2 in its trans state in water was observed to form nanoribbons. Nanotubes were obtained by irradiating an aqueous solution of 2 with UV light for $1 \mathrm{~h}$ post-sonication. The reverse isomerization (cis to trans) was obtained by heating the solution at $80^{\circ} \mathrm{C}$ for $1 \mathrm{~h}$ and cooling slowly to room temperature.

\section{S4. A note on vinylnitrile isomer naming conventions}

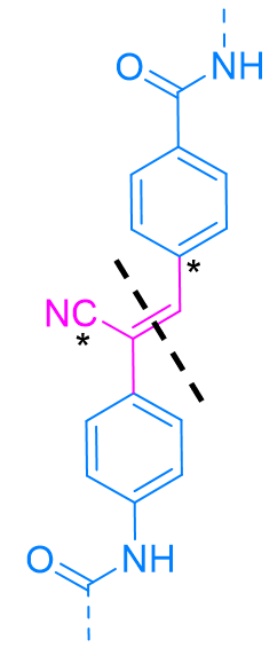

(Z) isomer

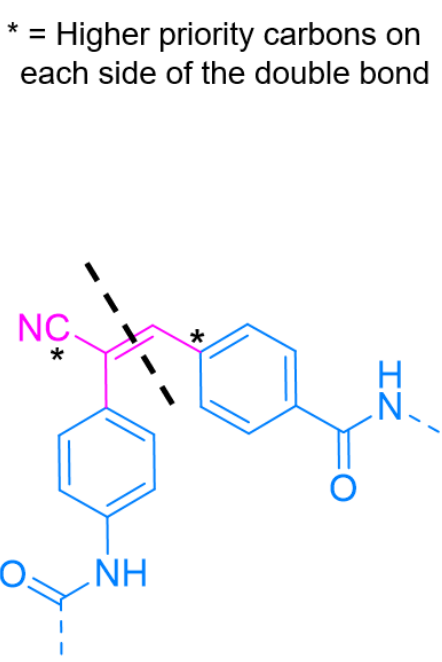

$(E)$ isomer

Figure S2. Naming of vinylnitrile isomers and identification of higher priority carbons on each side of the vinylnitrile double bond.

According to IUPAC naming conventions, the $(Z)$ isomer of cyanostilbene should be considered its cis isomer because the higher priority carbons fall on the "same side" of the double bond and the $(E)$ isomer should be considered the its trans isomer because the higher priority carbons fall on "opposite sides" of the double bond. However, the $(Z)$ and $(E)$ isomers are most frequently labelled in literature as trans and cis, respectively, because of unintended misnaming as an extension from stilbene isomer identification (e.g. the left molecule in Figure S2 would be trans if there were no substitutions on the double bond). For consistency with previous literature, we have chosen to use the colloquial nomenclature; that is, we denote the $(Z)$ isomer as trans and the $(E)$ isomer as cis. 


\section{S5. Characterization details}

Proton $\left({ }^{1} \mathrm{H}\right)$ and carbon $\left({ }^{13} \mathrm{C}\right)$ nuclear magnetic resonance (NMR) measurements were performed on a Bruker Avance III DPX 400 with samples dissolved in deuterated dimethylsulfoxide (DMSO- $d$ ). The chemical shifts were measured in parts per million (ppm) downfield from tetramethylsilane.

Molecular weight analysis of final compounds was conducted on a Bruker Omniflex matrix-assisted laser desportion/ionization-time of flight (MALDI-ToF). A matrix solution was prepared by adding $15 \mathrm{mg}$ of $\alpha$-cyano-4-hydroxycinnamic acid to $1 \mathrm{~mL}$ of $1: 1$ water:acetonitrile by volume with $0.1 \%$ TFA, vortexing for one minute, centrifuging for $20 \mathrm{~s}$, and retaining the supernatant. $10 \mu \mathrm{L}$ of a $1 \mathrm{mg} / \mathrm{mL}$ amphiphile solution was then transferred into a centrifuge tube and diluted with the matrix solution to a $50 \mathrm{pmol} / \mu \mathrm{L}$ concentration. $1 \mu \mathrm{L}$ of a $1 \mathrm{mg} / \mathrm{mL}$ calibrant solution (SpheriCal Peptide Low, Polymer Factory) in tetrahydrofuran was added to the solutions as an internal calibrant. $2 \mu \mathrm{L}$ of the final solution was pipetted and dried onto a sample plate for analysis.

Elemental analysis (EA) of final compounds was determined using a Vario EL elemental analysis (EA) instrument. Three replicate experiments were conducted per compound.

Ultraviolet-visible (UV-Vis) absorption spectra were captured on a JASCO V760 spectrophotometer. $365 \mathrm{~nm}$ wavelength UV light used to irradiate samples was produced by an LED Hönle 2.0 Powerpen. UV-Vis solutions were prepared at $0.1 \mathrm{mg} / \mathrm{mL}$.

Small angle X-ray scattering (SAXS) measurements were performed at Beamline 12ID-B of Advanced Photon Source at Argonne National Laboratory. The X-ray radiation energy was $13.3 \mathrm{keV}$ and a Pilatus $2 \mathrm{M}$ detector was employed for data collection. The 2-D X-ray scattering patterns were background subtracted and processed using beamline software for reduction to 1-D data curves. Amphiphile solutions at a $30 \mathrm{mg} / \mathrm{mL}$ concentration were used for SAXS measurements.

Transmission electron microscopy (TEM) images were captured on a FEI Tecnai G2 Spirit TWIN microscope at an accelerating voltage of $120 \mathrm{kV}$. Grids were prepared for TEM experiments by depositing a $7 \mathrm{mg} / \mathrm{mL}$ amphiphile solution onto a continuous carbon grid for $10 \mathrm{sec}$, blotting to remove the solution, depositing a $1 \%$ phosphotungstic acid solution onto the grid, and blotting to remove the stain. 
S6. Chemical characterization

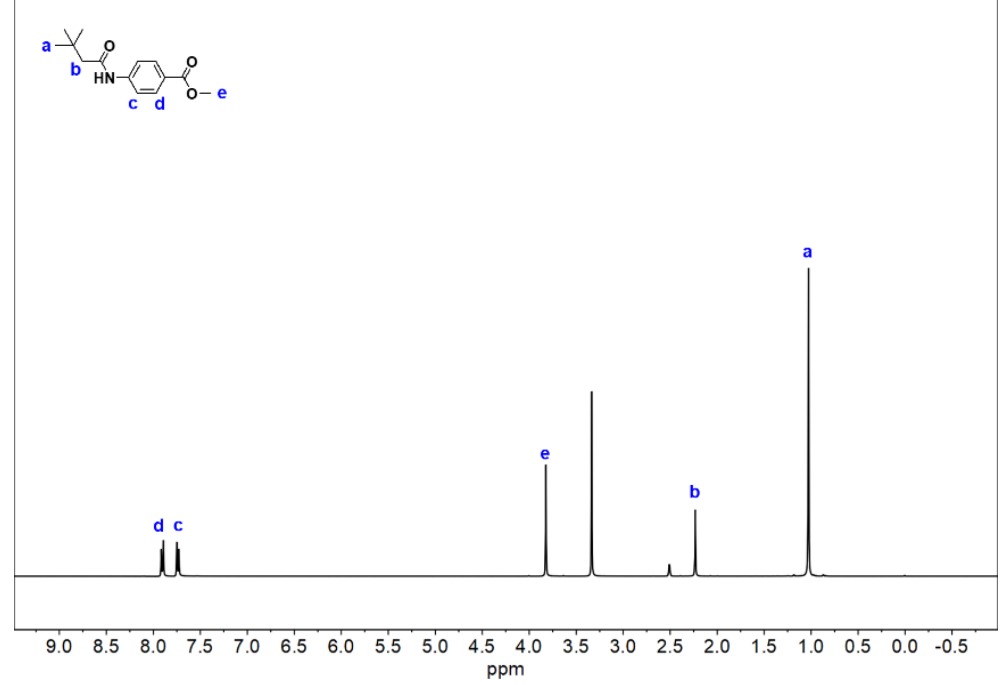

Figure S3. ${ }^{1} \mathrm{H}$ NMR spectra of compound A.

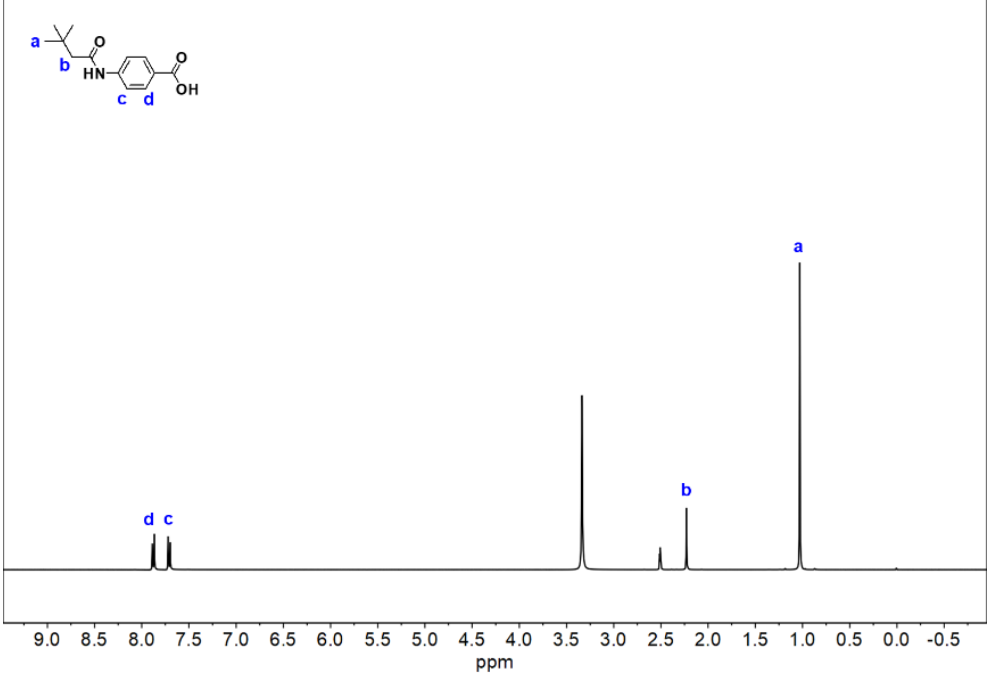

Figure S4. ${ }^{1} \mathrm{H}$ NMR spectra of compound $\mathbf{B}$. 


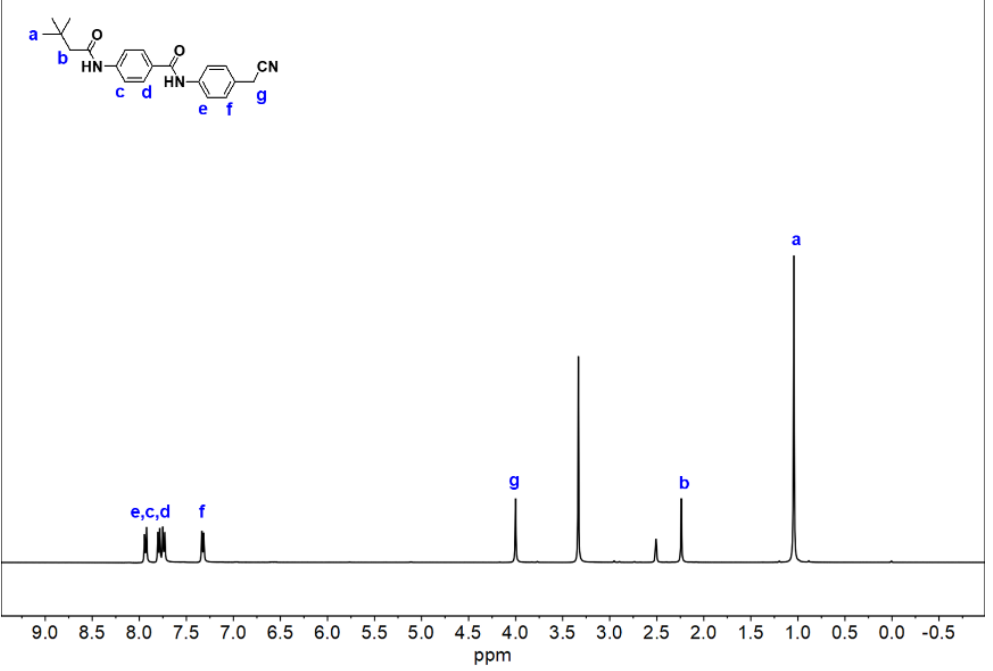

Figure S5. ${ }^{1} \mathrm{H}$ NMR spectra of compound $\mathbf{C}$.

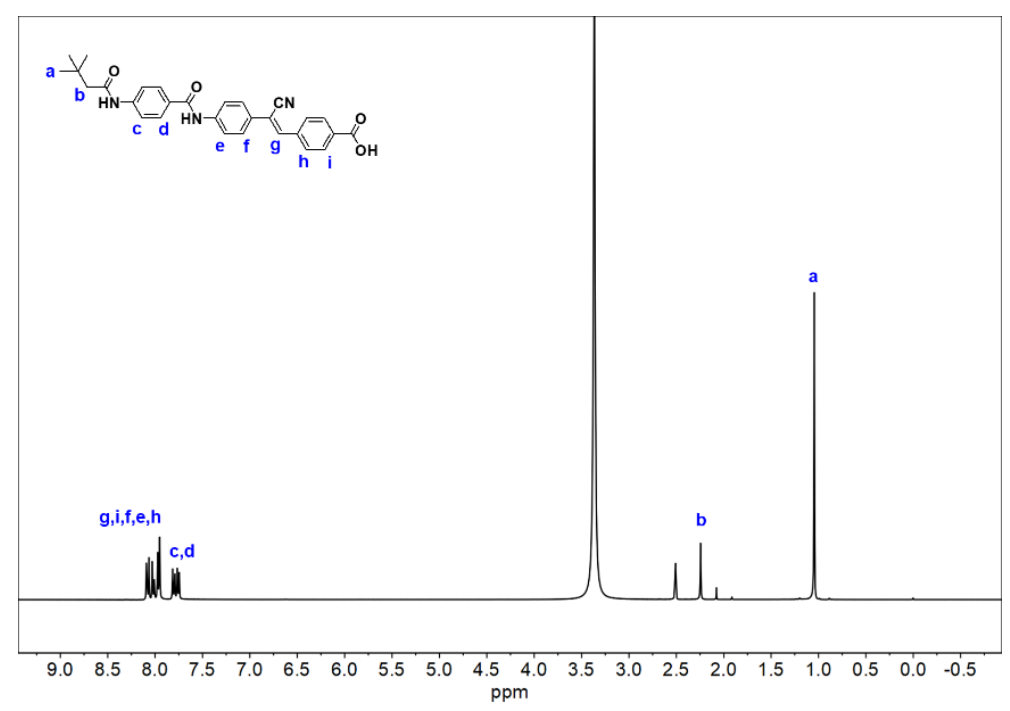

Figure S6. ${ }^{1} \mathrm{H}$ NMR spectra of compound D. 


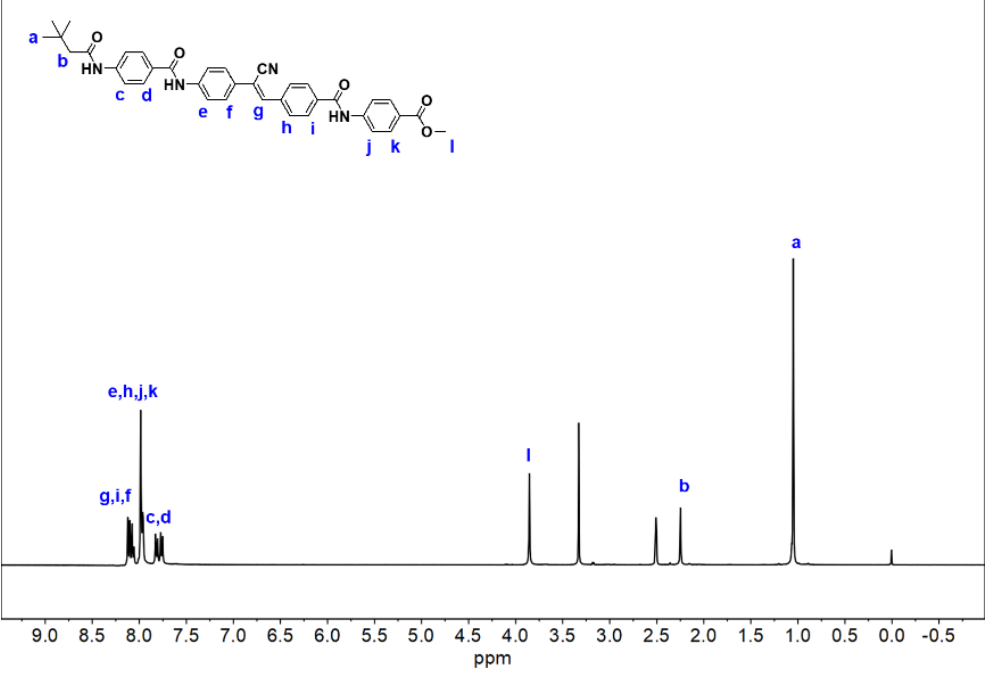

Figure S7. ${ }^{1} \mathrm{H}$ NMR spectra of compound $\mathbf{E}$.

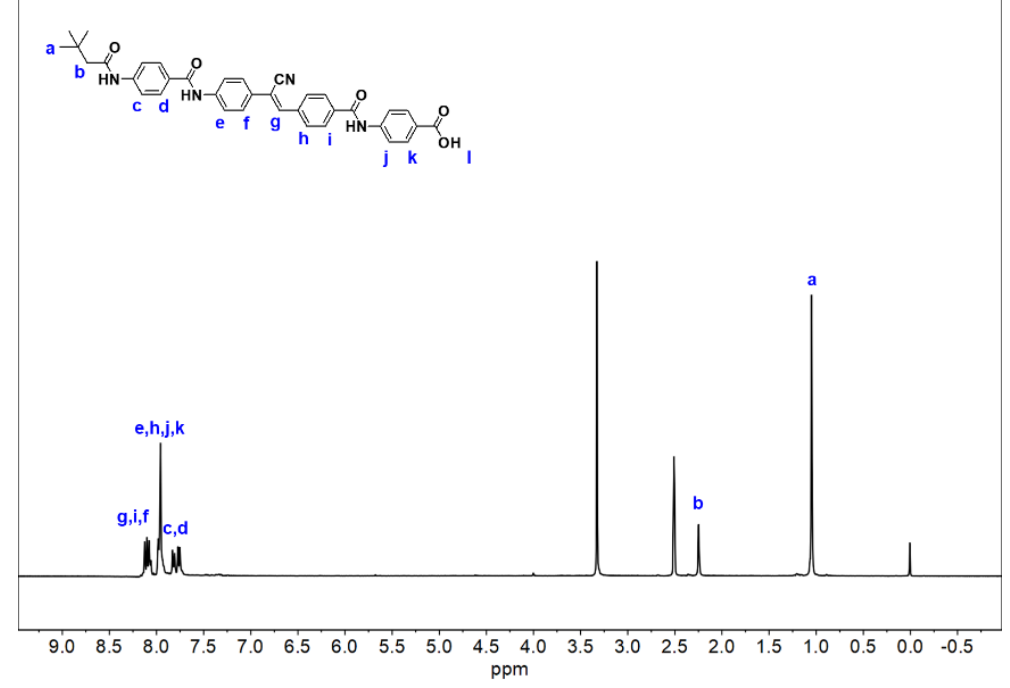

Figure S8. ${ }^{1} \mathrm{H}$ NMR spectra of compound $\mathbf{F}$. 


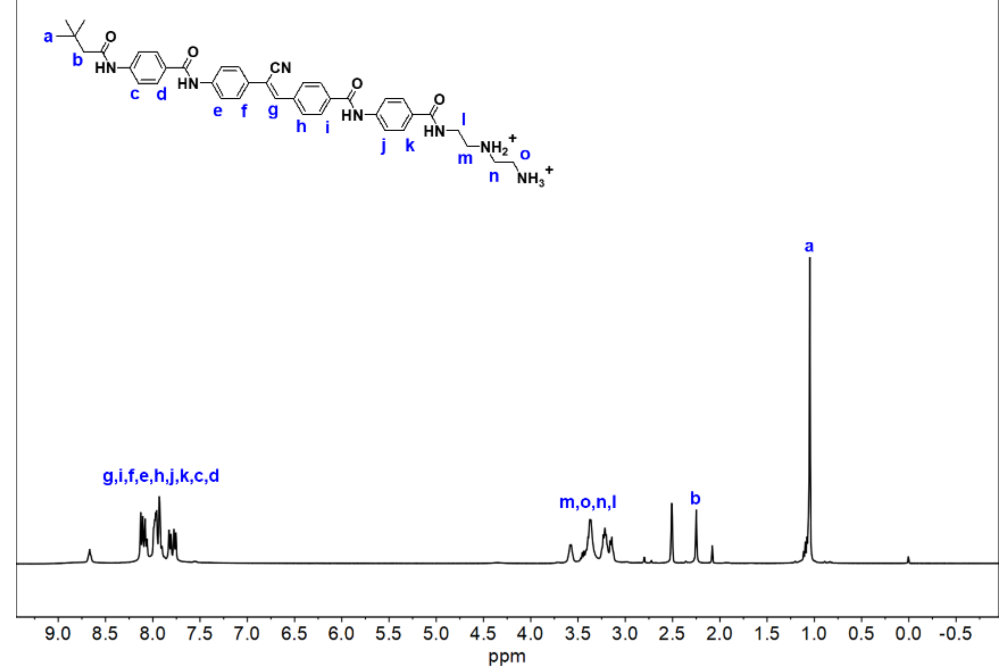

Figure S9. ${ }^{1} \mathrm{H}$ NMR spectra of 2.

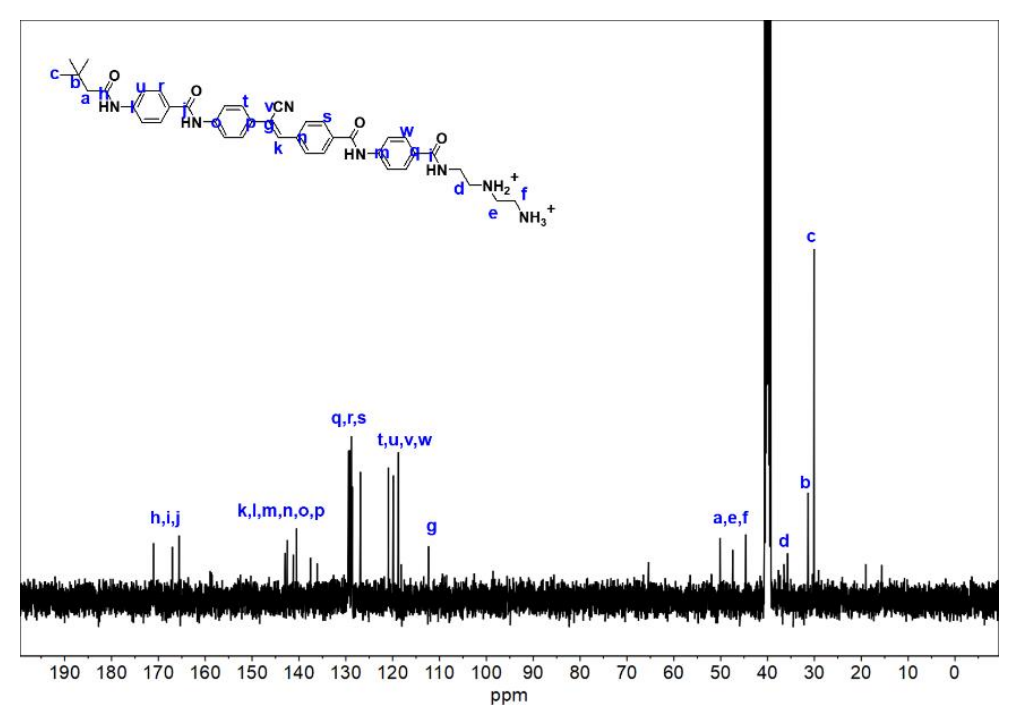

Figure S10. ${ }^{13} \mathrm{C}$ NMR spectra of 2 . 


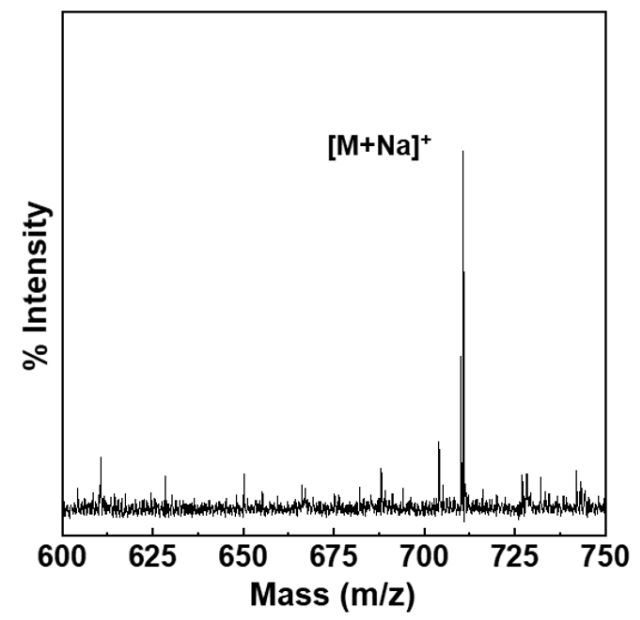

Figure S11. MALDI-ToF mass spectra of 2. The peak labelled $[\mathrm{M}+\mathrm{Na}]^{+}$corresponds to $\mathrm{m} / \mathrm{z}=710.83$, which matches the expected $\mathrm{m} / \mathrm{z}$ for $\mathbf{2}$ of 710.83 .

\begin{tabular}{|c|c|c|}
\hline & $\begin{array}{c}\text { Calculated } \\
\text { Content (wt \%) }\end{array}$ & $\begin{array}{l}\text { Experimental } \\
\text { Content (wt \%) }\end{array}$ \\
\hline \multirow{3}{*}{ C } & \multirow{3}{*}{69.85} & 69.84 \\
\hline & & 69.83 \\
\hline & & 69.81 \\
\hline \multirow{3}{*}{ H } & \multirow{3}{*}{6.59} & 6.57 \\
\hline & & 6.55 \\
\hline & & 6.51 \\
\hline \multirow{3}{*}{$\mathbf{N}$} & \multirow{3}{*}{14.25} & 14.24 \\
\hline & & 14.23 \\
\hline & & 14.19 \\
\hline
\end{tabular}

Figure S12. Elemental analysis of $\mathbf{2}$ over three replicate runs. 


\section{S7. Nanostructure fitting to small angle $X$-ray scattering profiles}

Fitting of SAXS data was completed in SasView software to determine the morphology of compound 1 and 2 nanostructures. Compound 1 was best fit to a lamellar_hg model, which provides separate scattering length densities for the head group and tail (including the structural domain) group. Compound $\mathbf{2}$ in its initial assembled state was best fit to a rectangular prism model; in its post-UV irradiation state was best fit to a hollow cylinder model; and in its postannealing state was best fit to a rectangular prism model. The following parameters were used for the fits.

\begin{tabular}{|c|c|}
\hline Model Parameter & Value \\
\hline Head Group Scattering Length Density & $9.40 \times 10^{-6} \AA^{-2}$ \\
\hline Tail Group Scattering Length Density & $12.6 \times 10^{-6} \AA^{-2}$ \\
\hline Solvent Scattering Length Density & $9.47 \times 10^{-6} \AA^{-2}$ \\
\hline Head Group Layer Total Thickness & $11.1 \AA$ \\
\hline Tail Group Layer Thickness & $28.0 \AA$ \\
\hline Background & $4.0 \times 10^{-5} \mathrm{~cm}^{-1}$ \\
\hline
\end{tabular}

Figure S13. Parameters for fitting SAXS of compound 1 nanostructures after initial assembly and after annealing to a lamellar model (Figure 2c).

\begin{tabular}{|c|c|}
\hline Model Parameter & Value \\
\hline Scattering Length Density & $9.98 \times 10^{-6} \AA^{-2}$ \\
\hline Solvent Scattering Length Density & $9.47 \times 10^{-6} \AA^{-2}$ \\
\hline Thickness & $47.0 \AA$ \\
\hline Width-to-thickness ratio & 1.15 \\
\hline Length-to-thickness ratio & 200.0 \\
\hline Distribution of width-to-thickness ratio & 1.13 \\
\hline Background & $5.0 \times 10^{-4} \mathrm{~cm}^{-1}$ \\
\hline
\end{tabular}

Figure S14. Parameters for fitting SAXS of compound 2 nanostructures after initial assembly to a rectangular prism model (Figure 4a). The distribution of width-to-thickness ratio polydispersity factor is defined as the fraction of the standard deviation of the width-tothickness ratio to its mean, and follows a Gaussian distribution. 


\begin{tabular}{|c|c|}
\hline Model Parameter & Value \\
\hline Scattering Length Density & $9.98 \times 10^{-6} \AA^{-2}$ \\
\hline Solvent Scattering Length Density & $9.47 \times 10^{-6} \AA^{-2}$ \\
\hline Core Radius & $97.0 \AA$ \\
\hline Shell Thickness & $60.7 \AA$ \\
\hline Length & $1000 \AA$ \\
\hline Background & $7.0 \times 10^{-6} \mathrm{~cm}^{-1}$ \\
\hline
\end{tabular}

Figure S15. Parameters for fitting SAXS of compound 2 nanostructures after UV irradiation to a hollow cylinder model (Figure 4c).

\begin{tabular}{|c|c|}
\hline Model Parameter & Value \\
\hline Scattering Length Density & $9.98 \times 10^{-6} \AA^{-2}$ \\
\hline Solvent Scattering Length Density & $9.47 \times 10^{-6} \AA^{-2}$ \\
\hline Thickness & $47.0 \AA$ \\
\hline Width-to-thickness ratio & 1.2 \\
\hline Length-to-thickness ratio & 200.0 \\
\hline Distribution of width-to-thickness ratio & 0.55 \\
\hline Background & $5.0 \times 10^{-4} \mathrm{~cm}^{-1}$ \\
\hline
\end{tabular}

Figure S16. Parameters for fitting SAXS of compound 2 nanostructures after annealing to a rectangular prism model (Figure 5c). The distribution of width-to-thickness ratio polydispersity factor is defined as the fraction of the standard deviation of the width-tothickness ratio to its mean, and follows a Gaussian distribution. 


\section{S8. Statistical topographical analysis of nanoribbon contours}

Sample preparation: A solution of compound 2 nanoribbons was prepared for atomic force microscopy (AFM) analysis by sonicating for $24 \mathrm{~h}$ at room temperature at $30 \mathrm{mg} / \mathrm{mL}$ and diluting to a final concentration of $0.03 \mathrm{mg} / \mathrm{mL}$. This solution was deposited onto a glass substrate which was cleaned with DI water and ethanol, dried with $\mathrm{N}_{2}(g)$, and activated with a UV/ozone treatment. The solution was incubated for $5 \mathrm{~min}$, and then rinsed off with DI water and dried with a $\mathrm{N}_{2}$ stream prior to AFM imaging.

AFM imaging: Images of compound 2 nanoribbons were captured in tapping mode in ambient air on a Bruker/JPK Nanowizard 4 AFM. Olympus AC160TS-R3 cantilevers were used, with a nominal spring constant of $26 \mathrm{~N} \mathrm{~m}^{-1}$ and a resonance frequency of approx. $300 \mathrm{kHz} 512 \mathrm{x}$ 512 pixel AFM images were produced at a 3-10 Hz scanning speed. A representative AFM image is shown in Fig. S17.

Statistical topographical analysis: Nanoribbon shape fluctuations were extracted from AFM images and statistically processed using Easyworm software. ${ }^{34}$ In this study, the software was used to produce parametric splines from the contours of $n=96$ nanoribbons. These parametric splines record coordinates of all knots along the ribbons, and each combination of knots is analyzed to obtain a secant length $L$ from which the midpoint of the secant deviates from the nanoribbon contour by the distance $\delta$. A persistence length $P$ is subsequently extracted via a least-squares fitting a worm-like chain model for semi-flexible polymers to the data, which relates the mean-square of $\delta$ to the secant length $L$ according to: $\left\langle\delta^{2}\right\rangle=L^{3} /(48 \times P)$. Thus, a higher persistence length corresponds to a smaller orientation change over a given distance along a nanoribbon's contour. The flexural rigidity $F$ is calculated using $F=P \times k_{B} T$, where the persistence length is scaled by thermal energy. Using this, the Young modulus $E$ is obtained by dividing the flexural rigidity by the nanoribbon's area moment of inertia, $E=F / I$. We use the AFM height of $\mathrm{d}=3.2 \pm 0.8 \mathrm{~nm}$ as the nanoribbon diameter in calculating the moment of inertia, $I=\pi \cdot d^{4} / 64$, as in our previous study. ${ }^{30}$ 


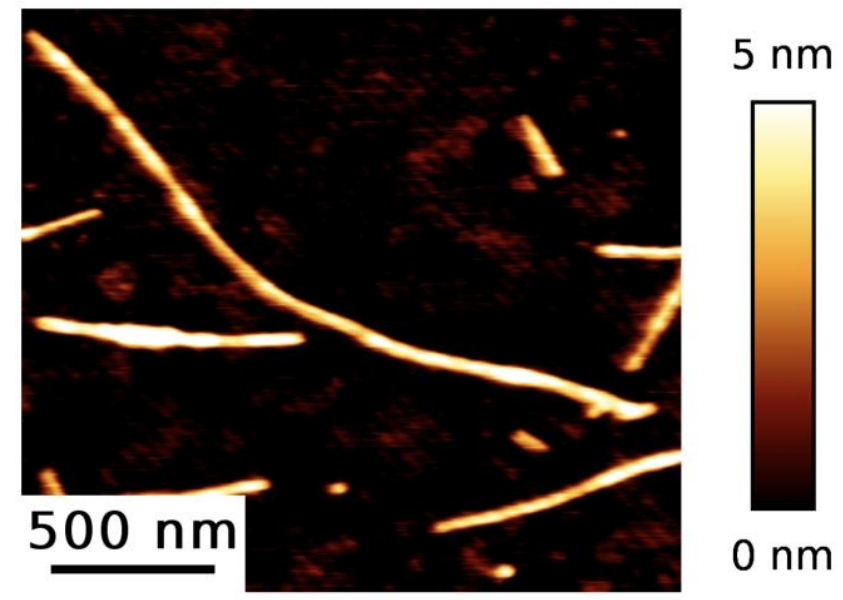

Figure S17. Representative AFM image of compound 2 nanoribbons used for statistical topographical analysis to determine nanoribbon stiffness. 


\section{S9. UV-Vis spectroscopy controls and experiments}

Compound 1, a conventional AA with no photoswitching group installed in the structural domain, shows no response to UVA irradiation for $1 \mathrm{~h}$, as shown in Figure S18.

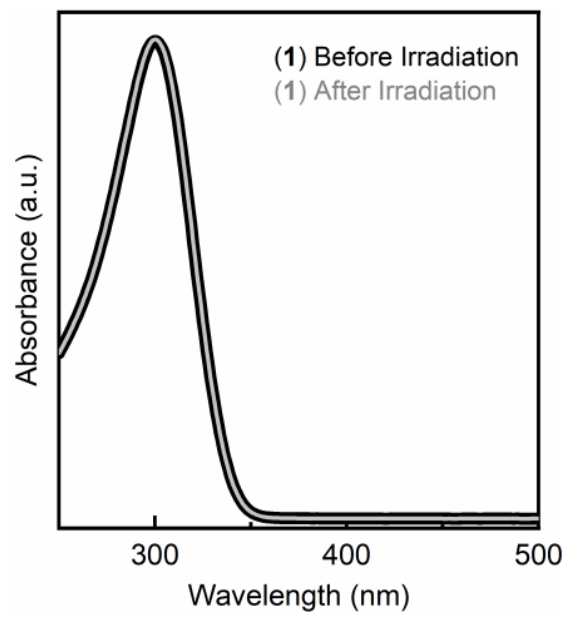

Figure S18. Compound 1 nanoribbon solution irradiated with

UVA light for $1 \mathrm{~h}$ shows no change in UV-Vis absorbance.
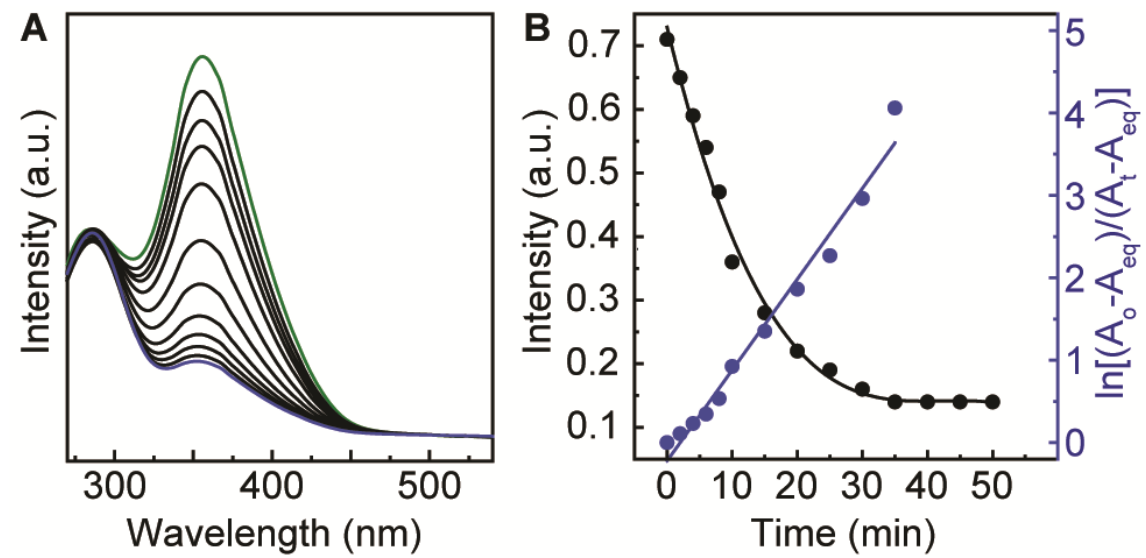

Figure S19. (a) UV-Vis spectra of compound 2 dissolved in DMSO with over 50 min of irradiation with UVA light irradiation. The initial state is the green line, and the final state is the blue line. (b) Time-dependent absorbance changes are used to calculate a photoisomerization rate constant of $K=1.10 \times 10^{-1} / \mathrm{min}$ of compound 2 in DMSO. 

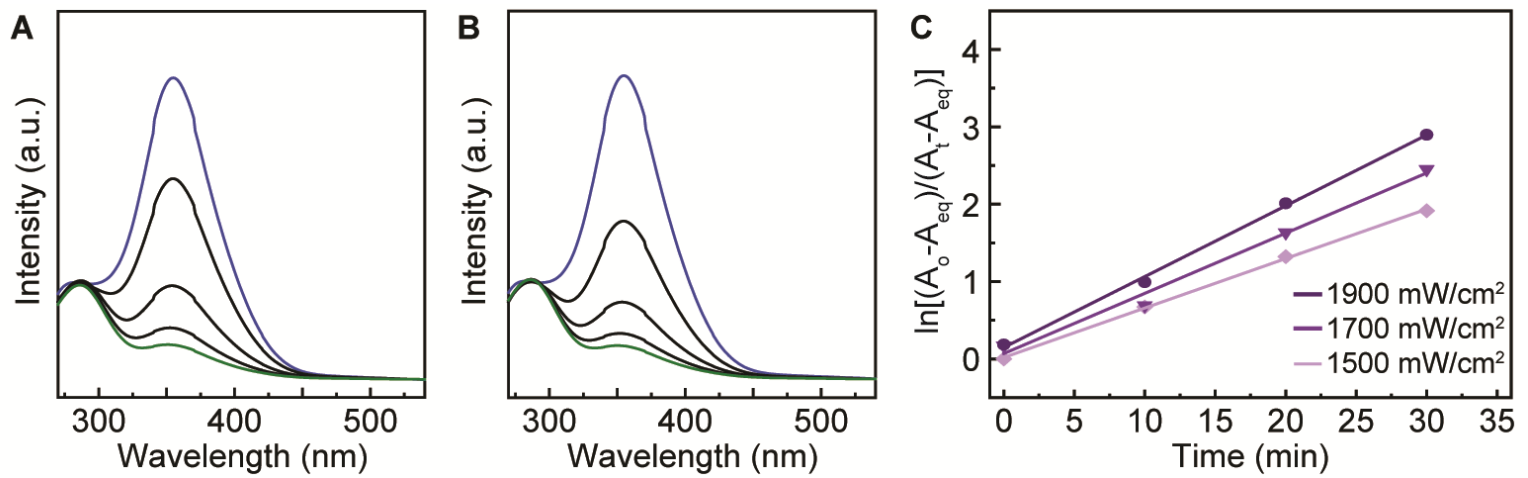

Figure S20. (a) Time-dependent UV-Vis spectra of compound 2 under medium intensity UVA light (1700 mW/cm²; initial line in blue, final line in green). (b) Time-dependent UVVis spectra of compound 2 under high intensity UVA light $\left(1900 \mathrm{~mW} / \mathrm{cm}^{2}\right.$; initial line in blue, final line in green). (c) From a first-order plot of the trans to cis isomerization of compound 2 at each irradiation power level, we extract photoisomerization rate constants of $5.44 \times 10^{-2} / \mathrm{min}, 7.77 \times 10^{-2} / \mathrm{min}$, and $9.15 \times 10^{-2} / \mathrm{min}$ for irradiation powers of $1500 \mathrm{~mW} / \mathrm{cm}^{2}, 1700 \mathrm{~mW} / \mathrm{cm}^{2}$, and $1900 \mathrm{~mW} / \mathrm{cm}^{2}$, respectively.
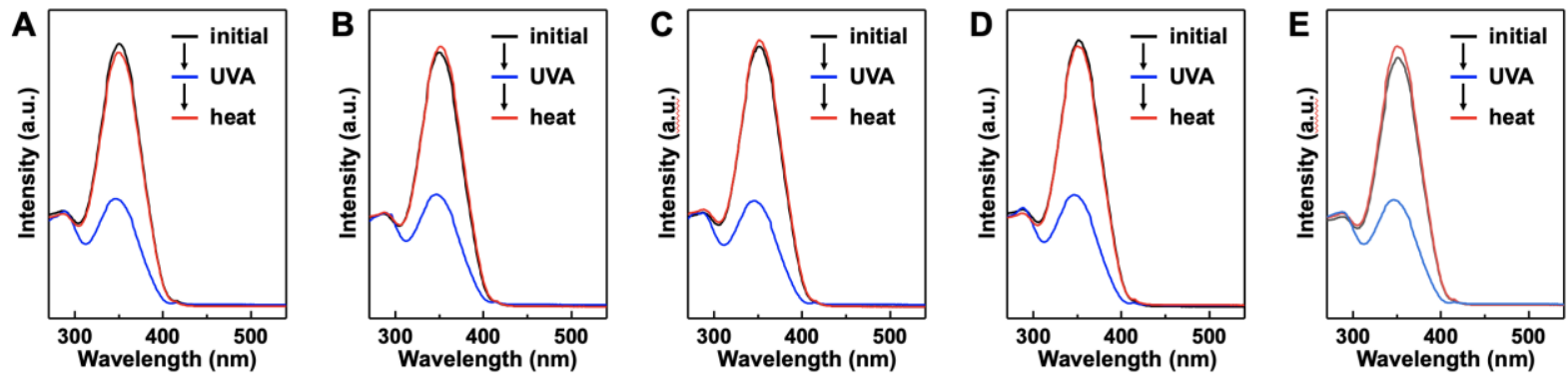

Figure S21. UV-Vis spectra of compound $\mathbf{2}$ in water initially, after UVA irradiation, and after annealing over five cycles: (a) cycle one, (b) cycle two, (c) cycle three, (d) cycle four, and (e) cycle five. The recovery of the absorbance to its initial state after annealing suggests that little to no photobleaching occurs during this process. 


\section{S10. Probing for the Tyndall effect in the trans and cis states of compound 2}

Atomic force microscopy (AFM) images which complement the investigation into the Tyndall effect in Figure S22 were recorded on a Park Systems NX10. A $7 \mathrm{mg} / \mathrm{mL}$ solution of 2 after self-assembly in water was diluted to 0.01 wt. $\%$, and then a $50 \mu \mathrm{L}$ droplet solution was deposited onto a freshly cleaned substrate. This substrate was prepared through a plane cleavage of mica, and cleaning with water several times. A PPP-NCHR non-contact cantilever was utilized to produce these images.

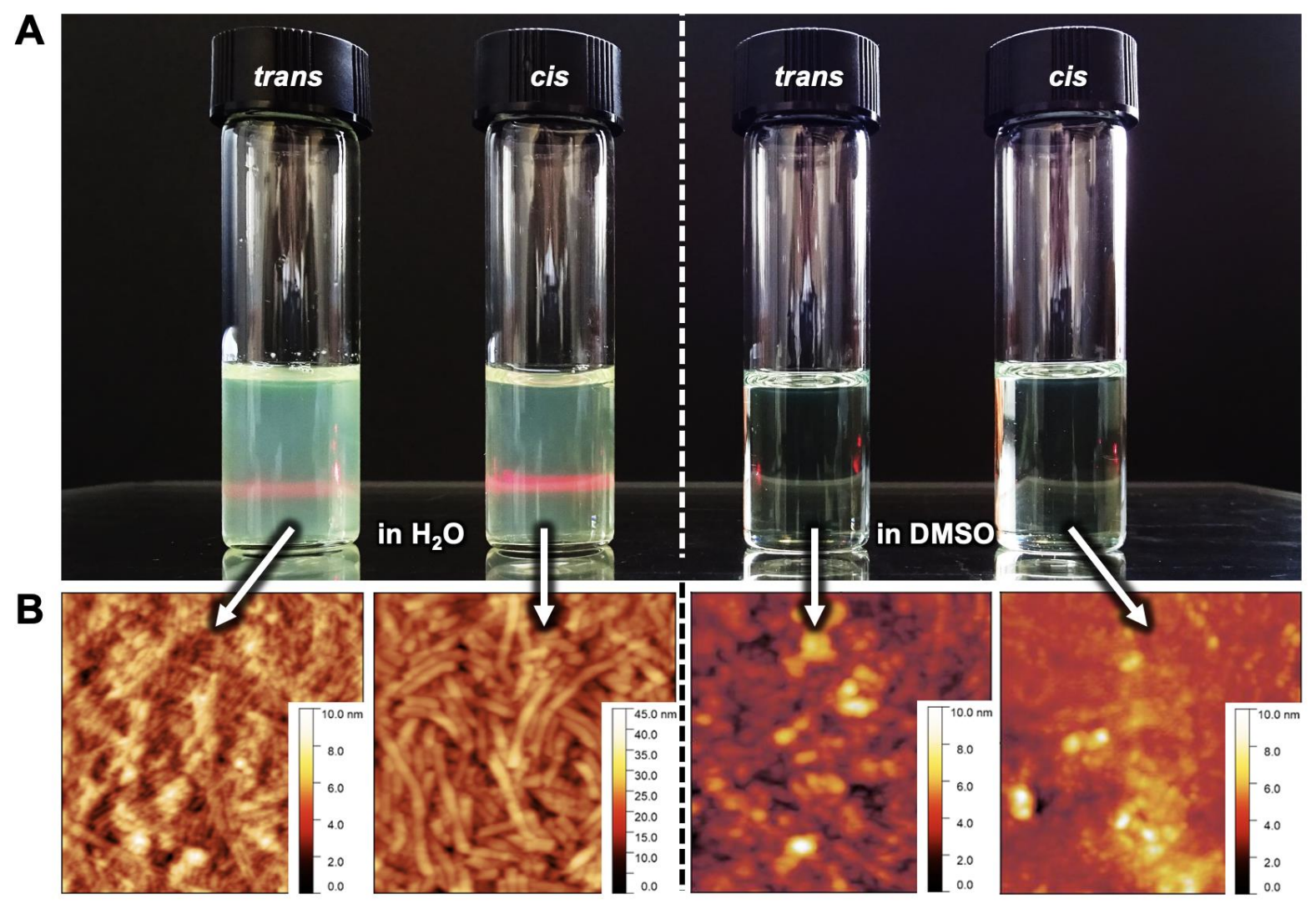

Figure S22. (a) Observation of the Tyndall effect of $7 \mathrm{mg} / \mathrm{mL}$ solutions of compound 2 in water (left two vials) or DMSO (right two vials) in its trans or cis state. The scattering observed by compound $\mathbf{2}$ in water is indicative of the maintenance of a nanostructure; its absence in DMSO indicates that compound 2 does not form an ordered nanostructure. (b) AFM micrographs of the solutions from each vial indicate show, from left to right, nanoribbons from the assembly of compound $\mathbf{2}$ in water in its trans state; nanotubes following the irradiation of compound $\mathbf{2}$ in water into a cis-rich state; and no ordered nanostructure when compound $\mathbf{2}$ is dissolved in DMSO in either its trans or cis states. 


\section{S11. Packing of compound 2 into nanoribbons and nanotubes}

One dimensional (1D) wide-angle X-ray diffraction (WAXD) experiments were conducted on a Bruker D8 Discover using $\mathrm{Cu} \mathrm{K} \alpha$ radiation. The diffraction peak positions and widths were calibrated with crystalline silicon powder. Background scattering was subtracted from the samples using instrument software. $30 \mathrm{mg} / \mathrm{mL}$ solutions of compound 2 nanoribbons and nanotubes were lyophilized for WAXD analysis.

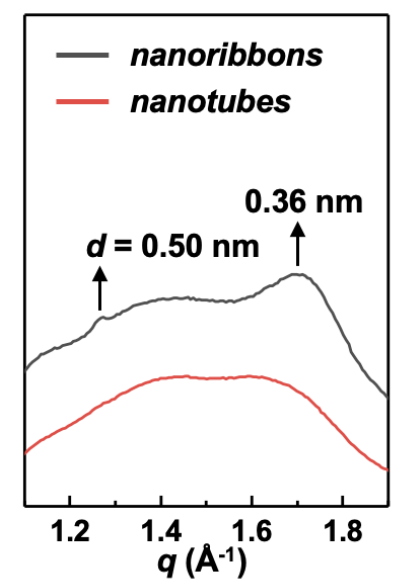

Figure S23. 1D WAXD pattern of lyophilized compound 2 nanoribbons and nanotubes. The geometries of these nanostructures are observed to retain their shape when dried by AFM. 